\title{
Interférences
}

Ars scribendi

$8 \mid 2015$

L'exil au miroir de la Correspondance de Cicéron

\section{Lettres et discours de Cicéron : regards croisés sur les années 58-56}

\section{Gérard Salamon}

\section{(2) OpenEdition \\ 12 Journals}

Édition électronique

URL : http://journals.openedition.org/interferences/5496

DOI : 10.4000/interferences.5496

ISSN : $1777-5485$

Éditeur

HiSoMA - Histoire et sources des Mondes antiques

Référence électronique

Gérard Salamon, "Lettres et discours de Cicéron : regards croisés sur les années 58-56 ", Interférences [En ligne], 8 | 2015, mis en ligne le 31 octobre 2014, consulté le 10 décembre 2020. URL : http:// journals.openedition.org/interferences/5496; DOI : https://doi.org/10.4000/interferences.5496

Ce document a été généré automatiquement le 10 décembre 2020.

Tous droits réservés 


\title{
Lettres et discours de Cicéron : regards croisés sur les années 58-56
}

\author{
Gérard Salamon
}

1 Ce que nous sommes convenus d'appeler l'exil de Cicéron, même si lui-même n'emploie jamais ce terme dans sa correspondance et le récuse dans ses discours ${ }^{1}$, a bien entendu totalement interrompu sa carrière d'orateur. Dans les lettres de l'année 58, il présente cette carrière comme appartenant à un passé révolu et y voit même la cause directe du malheur qui les accable, lui et les siens, comme il l'écrit à Terentia (Cic., Fam. 14, 2, 2) :

Hem, mea lux, meum desiderium, unde omnes opem petere solebant! te nunc, mea Terentia, sic uexari, sic iacere in lacrimis et sordibus, idque fieri mea culpa, qui ceteros seruaui, ut nos periremus $^{2}$.

Hélas, lumière de ma vie, objet de mon regret, toi à qui tous avaient coutume de demander de l'aide ! Te voilà maintenant, ma chère Terentia, ainsi malmenée, ainsi plongée dans les larmes et le deuil, et cela par ma faute, à moi qui ai sauvé tous les autres au prix de ma propre perte.

Mais cette carrière reprend dès qu'il est de retour à Rome, le 4 septembre 57 : quelques jours plus tard, il écrit à Atticus qu'il a en effet aussitôt recouvré - en dépit de ses craintes - à la fois son auctoritas et son statut d'orateur de premier plan (splendor noster ille forensis) ${ }^{3}$. Le fait est que la correspondance des années 57 et 56 témoigne d'une intense activité oratoire. La lettre à Atticus que je viens de citer mentionne, par exemple, trois discours ${ }^{4}$ : le discours de remerciements au Sénat (Cum Senatui gratias agit), daté du 5 septembre, ainsi que deux discours prononcés le 7 septembre et adressés l'un au Sénat pour le convaincre d'attribuer à Pompée la préfecture de l'annone, l'autre au peuple ${ }^{5}$. Une nouvelle lettre à Atticus, datée de la première moitié d'octobre, évoque le De domo sua, prononcé le 29 septembre : Cicéron est manifestement très satisfait de ce discours, puisqu'il demande à son ami de le diffuser afin de ne pas priver les jeunes gens d'une occasion d'apprécier son éloquence ${ }^{6}$. Une lettre du 10 décembre adressée à Quintus mentionne ensuite un long discours qu'il a prononcé au Sénat contre Clodius : à l'en croire, il a été bien accueilli ${ }^{7}$. Le 15 janvier 56 , Cicéron informe Cornelius Lentulus Spinther que deux jours plus tôt il a longuement parlé en sa faveur au Sénat à propos de la question d'Égypte avec, selon lui, un certain succès ${ }^{8}$. 
Nous apprenons ensuite, par deux lettres à Quintus datées respectivement de la mifévrier et de la mi-mars 56, qu'il a défendu d'abord L. Bestia dans un procès de ambitu 9 , puis - sans doute dans un procès de ui-P. Sestius dont il se félicite qu'il ait été acquitté; il mentionne à cette occasion un autre de ses discours, l'Interrogatio in Vatinium ${ }^{10}$. Mais ce ne sont pas les seuls procès dans lesquels Cicéron intervient en cette période, comme en témoigne - dans la lettre suivante à Quintus - la formule in iudiciis sumus qui fuimus; domus celebratur ita ut cum maxime ${ }^{11}$. Dans le développement qui suit, il se réjouit de voir condamnés tous ses ennemis personnels (inimici), à l'exception de Sex. Clodius, sans que l'on puisse savoir précisément s'il plaide ou non en personne dans les procès en question ${ }^{12}$. Le dernier discours dont la correspondance de 56 fasse mention, dans une lettre à Atticus datant du mois de juin, est le De prouinciis consularibus, que Cicéron présente, on le sait, comme une palinodie quelque peu honteuse et qui marque son rapprochement avec le triumvirat ${ }^{13}$.

3 La liste ainsi établie suscite deux remarques complémentaires l'une de l'autre. La première est qu'elle contient des discours qui ne nous sont pas parvenus, soit qu'ils n'aient pas été publiés, soit qu'ils aient été perdus. C'est le cas par exemple du plaidoyer en faveur de Bestia, du discours destiné à appuyer les revendications de Cornelius Lentulus Spinther dans l'affaire d'Égypte et enfin de ceux qu'il prononça contre Clodius ou en faveur de Pompée. Pourtant ce dernier discours valut manifestement à l'orateur, $\mathrm{y}$ compris dans son camp, un certain nombre de critiques, auxquelles répond tout le début du De domo sua, alors même que la question est sans lien direct avec le sujet ${ }^{14}$. La deuxième remarque est tout aussi intéressante : sont en effet absents de cette liste des discours prononcés à la même période et que nous avons conservés : c'est le cas du Pro Caelio mais aussi du De haruspicum responso, prononcé au Sénat à la suite d'une consultation des haruspices dans la réponse desquels Clodius avait vu une occasion de remettre en cause la décision prise l'année précédente de rendre à Cicéron son terrain et de lui permettre d'y reconstruire sa maison ${ }^{15}$.

4 Il est évidemment impossible d'aborder ici tous les discours de la période et tous les aspects de la question des rapports qu'ils entretiennent avec la Correspondance. J'ai donc fait le choix de me limiter à six discours : les deux discours de remerciements (au Sénat et au peuple), le De domo sua, le Pro Sestio, l'Interrogatio in Vatinium, et enfin le De haruspicum responso. Ces discours sont prononcés devant des auditoires différents (le Sénat, le peuple, le tribunal des Pontifes, celui du préteur), en des occasions et à des moments différents (les trois premiers en 57 juste après le retour de Cicéron, les trois autres en 56); mais ils ont en commun de permettre à l'orateur de revenir longuement sur l'histoire de la période récente, c'est-à-dire sur son histoire, ce qu'il appelle luimême dans la lettre à Lucceius la fabula rerum euentorumque nostrorum ${ }^{16}$. En bref, ces six discours constituent à des titres divers ce que l'on appellerait de nos jours, dans le langage journalistique, un story telling ${ }^{17}$, qui se construit souvent par le détournement de l'objet annoncé du discours. C'est évident dans le Pro Sestio où Cicéron, au prétexte que Sestius est attaqué d'abord parce qu'il est l'un de ses partisans ${ }^{18}$, parle surtout de lui-même, ce qui lui a souvent été reproché ${ }^{19}$; mais c'est aussi le cas dans le De domo sua où, reconnaissant sans ambages qu'il multiplie les digressions ${ }^{20}$, Cicéron n'aborde la question de la consécration de sa maison que dans les toutes dernières pages ${ }^{21}$. C'est le discours que Cicéron tient sur lui-même qu'il me paraît intéressant de comparer avec l'image qu'il donne de lui dans la Correspondance: j'aborderai donc ici deux aspects, indissociables comme je voudrais le montrer, de cette histoire reconstruite en étudiant 
ce que les discours disent d'abord des raisons qui ont conduit Cicéron à quitter Rome, ensuite de la nature exacte de ce qu'il définit comme son " éloignement ».

Dans la Correspondance, lorsque Cicéron évoque sa décision de quitter Rome, ce qu'il fait à de très nombreuses reprises, c'est toujours pour se reprocher d'avoir manqué à son devoir et de l'avoir prise alors qu'il avait d'autres solutions, qu'il détaille dans une lettre à Terentia du 28 novembre 58 : accepter la légation que lui offrait César, résister à Clodius ou encore mourir en brave ${ }^{22}$. En guise de justification, ou tout au moins d'explication, il donne à son départ de multiples raisons: la trahison de ceux qu'il croyait ses amis et qui ont fait passer leur intérêt avant le sien par peur ou par envie ${ }^{23}$, les mauvais conseils qui lui ont été donnés et qu'il a suivis au lieu de s'en tenir à son propre sentiment ${ }^{24}$, l'absence de réel soutien de la part d'Atticus ${ }^{25}$, les larmes des siens qui l'ont forcé à vivre ${ }^{26}$, enfin sa propre peur ${ }^{27}$ et son aveuglement qui l'ont empêché d'analyser sainement la situation alors qu'il n'avait rien à craindre de la première loi de Clodius qui ne le visait pas nommément ${ }^{28}$. En bref, comme en témoigne dans une sorte de résumé saisissant, en raison de la récurrence qu'on y observe des formes passives, une lettre à Atticus du 17 août 58, la Correspondance donne l'image d'un homme dont la faute (culpa ou error) a été de subir les événements en perdant toute prise sur eux ${ }^{29}$. Faute d'un jugement sûr, il a donc été conduit, écrit-il, à prendre le parti qui était sans conteste le plus honteux de tous : hoc miserius, turpius, indignius nobis nihil fuit ${ }^{30}$.

6 Les discours donnent une version bien différente des faits puisque Cicéron y présente son départ comme résultant non pas d'une décision contrainte, mais tout au contraire d'un choix délibéré, celui de faire passer le salut de l'État avant son propre salut. Le motif (au sens littéraire du terme) apparaît dans le premier des discours qu'il prononce après son retour, le Discours de remerciements au Sénat, où l'année 58 est dès l'abord évoquée par la formule : ille annus quem ego mihi quam patriae malueram esse fatalem ${ }^{31}$. Il fait ensuite, juste avant la péroraison, l'objet d'un long développement, très précisément argumenté et que l'on peut résumer comme suit ${ }^{32}:$ j'aurais pu, comme beaucoup d'hommes de cœur me le conseillaient, combattre Clodius, ce nouveau Catilina, par les armes, mais je savais que ma victoire ne ferait que me susciter de nouveaux ennemis et que ma défaite entraînerait la mort de beaucoup d'hommes de bien; je n'ai pas pu m'y résoudre et employer, alors que je n'étais plus qu'un simple particulier (priuatus), la violence à laquelle j'avais refusé de recourir comme consul ${ }^{33}$; quant au suicide, il n'aurait été envisageable que si j'avais été persuadé en partant de ne jamais être rappelé, ce qui était évidemment impossible ne serait-ce qu'à imaginer ; mon départ était donc la meilleure solution pour éviter un bain de sang, d'autant que je ne pouvais rester à Rome quand la République elle-même en était bannie ${ }^{34}$.

7 Ce passage du Discours de remerciements au Sénat est particulièrement révélateur de ce qui se joue alors : en effet, la lettre à Atticus qui le mentionne à un moment où l'on peut penser qu'il est connu de tous commence par un rappel de la thématique des lettres d'exil, construite autour de l'imprudentia et du triptyque error, furor, timor ${ }^{35}$. La comparaison entre la lettre et le discours fait évidemment apparaître la version des événements présentée dans celui-ci comme une réécriture de l'histoire, et cela de façon tellement évidente pour Atticus que l'on peut se demander si la lettre n'a pas précisément pour fonction de signifier à celui-ci que le chapitre est clos et qu'il convient désormais de passer à autre chose. Mais si l'on peut légitimement accuser Cicéron de tenir sur les raisons de son départ de Rome un double langage, il faut se garder d'opposer un langage de vérité, qui serait celui des lettres d'exil, à une 
réécriture falsificatrice, qui serait celle des discours, et cela pour deux raisons complémentaires. La première est qu'on sait bien que l'image que Cicéron donne de lui dans les lettres d'exil tient largement de la " posture » et qu'elle vise avant tout à agir sur ses correspondants pour qu'ils interviennent en sa faveur. La seconde est que la version des faits présentée dans les discours n'est pas, au moins pour l'un de ses éléments de base, pure fiction : toute une série de lettres à Atticus et à Quintus datées de la fin de l'année 59 attestent que Cicéron a réellement envisagé pendant un moment - même s'il le dit peut-être pour tester ses éventuels soutiens - de s'opposer par les armes à Clodius avec l'appui de ce qu'il définit comme l'armée des boni ${ }^{36}$. C'est bien entendu moins par volonté de ne pas nuire à l'État qu'en raison du faible soutien dont il a alors bénéficié de la part de ses amis politiques qu'il a décidé de ne pas engager la lutte contre Clodius, mais la réécriture est moins radicale qu'on ne l'a souvent dit. En tout état de cause, une fois énoncé le motif du sacrifice personnel pour le bien de l'État, Cicéron s'y tient et ne manque jamais de le rappeler dans les discours de cette période, y compris lorsque rien ne l'impose comme c'est le cas par exemple dans l'Interrogatio in Vatinium ${ }^{37}$.

8 Le Discours de remerciements au peuple, prononcé deux jours plus tard, est encore plus explicite, puisque dès l'exorde Cicéron présente la décision qu'il a prise de quitter Rome comme une deuotio (Cic., Red. Pop. 1) :

Quod precatus a Ioue Optimo Maximo ceterisque dis immortalibus sum, Quirites, eo tempore cum me fortunasque meas pro uestra incolumitate otio concordiaque deuoui, ut, si meas rationes umquam uestrae saluti anteposuissem, sempiternam poenam sustinerem mea uoluntate susceptam, sin et ea quae ante gesseram conseruandae ciuitatis causa gessissem et illam miseram profectionem uestrae salutis gratia suscepissem, ut quod odium scelerati homines et audaces in rem publicam et in omnis bonos conceptum iam diu continerent, id in me uno potius quam in optimo quoque et uniuersa ciuitate defigerent, hoc si animo in uos liberosque uestros fuissem, ut aliquando uos patresque conscriptos Italiamque uniuersam memoria mei misericordia desideriumque teneret, eius deuotionis me esse conuictum iudicio deorum immortalium, testimonio senatus, consensu Italiae, confessione inimicorum, beneficio diuino immortalique uestro maxime laetor.

Étant donné que j'ai imploré Jupiter très bon et très grand et tous les autres dieux immortels, citoyens, au moment où j'ai dévoué ma personne et mes biens pour votre sauvegarde, votre repos et votre concorde en demandant que, si jamais j'avais préféré mes intérêts à votre salut, ils m'infligent une peine éternelle subie de ma propre volonté, mais que, si mes actions antérieures avaient eu pour but la conservation de la cité et si j'avais enduré ce triste départ pour votre salut, afin de détourner la haine que des hommes, scélérats et pleins d'audace, nourrissaient depuis longtemps contre l'État et tous les gens de bien en la fixant sur moi plutôt que sur tous les meilleurs et la cité tout entière, si donc j'avais eu ces sentiments envers vous et vos enfants, qu'un jour ils vous donnent, ainsi qu'aux pères conscrits et à l'Italie tout entière, le souvenir, la pitié et le regret de ma personne, je me réjouis énormément que cet acte de dévotion ait été sanctionné par le jugement des dieux immortels, le témoignage du Sénat, l'accord de l'Italie, l'aveu de mes ennemis, votre bienfait divin et immortel.

9 Dans la mesure où une deuotio est au sens strict « le sacrifice qu'un général fait de sa vie sur le champ de bataille pour sauver son armée de la défaite et de l'anéantissement ", l'emploi du terme par Cicéron apparaît ici au mieux comme une métaphore hyperbolique, au pire comme le détournement d'un rituel dont l'efficacité est assurée par la mort, aux yeux de tous, de celui qui l'exécute ${ }^{38}$. L'explication selon laquelle Cicéron pourrait se permettre sans risque un tel détournement dans un discours au peuple, censé être peu au fait - ce qui d'ailleurs resterait à prouver - du sens réel des 
mots, n'est pas satisfaisante ${ }^{39}:$ car, si le terme est effectivement absent du Discours de remerciements au Sénat, il est repris dans la péroraison du De domo sua ${ }^{40}$, qui est prononcé devant les pontifes, auditoire qu'on peut difficilement soupçonner de ne pas connaître le sens du terme. D'ailleurs, dans le corps même du discours, Cicéron assume totalement, serais-je tenté de dire, le détournement qu'il fait du terme : il prend en effet comme exemple les Decii, qui sont assurément les spécialistes incontestés en la matière ${ }^{41}$, mais souligne qu'il avait d'autant moins hésité à les imiter que sa deuotio pro salute uniuersae rei publicae à lui, puisqu'elle consistait non à mourir, mais à vivre, avait l'immense avantage de lui permettre d'être, une fois revenu à Rome, « le spectateur de sa propre gloire ${ }^{42}$.

Une telle interprétation du rituel ne résistait évidemment pas à l'analyse et ne pouvait être longtemps maintenue. La preuve en est que dans le Pro Sestio, où le motif réapparaît, il fait l'objet d'un traitement plus traditionnel ${ }^{43}$ : la deuotio, le mot étant pris cette fois dans son sens strict, n'y est plus envisagée que comme une solution que Cicéron aurait adoptée sans hésiter si la situation avait été telle que par sa mort il avait pu sauver la République ${ }^{44}$. Le verbe deuoueo n'est employé que pour les Decii qui apparaissent eux-mêmes au sein d'une liste plus large de Romains morts pour la patrie, sans qu'on puisse parler dans leur cas de deuotio ${ }^{45}$. Mais en l'occurrence, poursuit Cicéron, sa mort, loin de sauver l'État, aurait condamné celui-ci à périr en le privant du seul homme qui était prêt à le défendre au prix de son propre malheur; c'est au contraire en restant en vie, donc par son départ, qu'il l'avait préservé en détournant sur lui-même les coups qui allaient frapper la République ${ }^{46}$.

11 D'un discours à l'autre, la présentation que fait Cicéron du motif du sacrifice de sa personne à l'État varie donc assez sensiblement, mais c'est toujours la même idée qu'il s'efforce d'imposer. Il s'agit pour lui de se présenter comme ayant sauvé deux fois la République, d'abord lors de son consulat de 63 en mettant fin à la conjuration de Catilina, ensuite en 58 en choisissant de quitter Rome, et dans les deux occasions sans recourir à la lutte armée. C'est l'idée qu'il développe dans le De domo sua lorsqu'il prend à parti Clodius ${ }^{47}$, et que résume la formule du Pro Sestio qui clôt le passage que j'ai cité plus haut: unus rem publicam bis seruaui, semel gloria, iterum aerumna mea ${ }^{48}$.

Une telle réécriture imposait bien entendu de régler une fois pour toutes le problème juridique posé par les conditions dans lesquelles s'était fait le départ de Cicéron, que ses adversaires présentaient comme un exil, ultime recours autorisé, on le sait, aux Romains pour échapper à une condamnation. Une telle présentation des choses revenait évidemment à réduire à néant l'ensemble de l'argumentation développée par Cicéron dans ses discours. La question est, à vrai dire, complexe, mais, du point de vue du droit, c'est la position de Clodius qui semble avoir été mal assurée puisque si sa deuxième loi, celle qui condamnait Cicéron à résider à 500 milles au moins des côtes de l'Italie, était considérée comme un priuilegium, elle pouvait, à ce titre, être purement et simplement frappée de nullité. Dans le De domo sua, Cicéron attaque de front Clodius sur cette question, d'abord en saisissant la première opportunité qui lui est offerte (en l'occurrence le reproche que lui a adressé Clodius d'avoir fait voter en faveur de Pompée une mesure extra ordinem) pour dénoncer sa loi comme illégale ${ }^{49}$, ensuite en faisant de cette loi une analyse extrêmement détaillée ${ }^{50}$ et fort instructive en matière de droit romain ${ }^{51}$. Dans le cadre du discours, un tel développement s'explique aisément puisque c'est sur sa loi que Clodius s'appuie pour affirmer que la consécration qu'il a faite du terrain de Cicéron à la liberté est on ne peut plus légale, donc irrévocable. Mais, 
de manière plus générale, c'est l'occasion pour Cicéron d'insister sur le fait qu'il a quitté Rome sans même avoir été cité en justice ${ }^{52}$ et qu'il ne peut donc en aucun cas être considéré comme un exilé (Cic., Dom. 72) :

Hunc tu etiam, portentosa pestis, exsulem appellare ausus es, cum tantis sceleribus esses et flagitiis notatus ut omnem locum quo adisses exsili simillimum redderes? Quid est enim exsul ? ipsum per se nomen calamitatis, non turpitudinis. Quando igitur est turpe? re uera, cum est poena peccati, opinione autem hominum etiam, si est poena damnati. Vtrum igitur peccato meo nomen subeo an re iudicata? Peccato? Iam neque tu id dicere audes, quem isti satellites tui «felicem Catilinam » nominant, neque quisquam eorum qui solebant. Non modo iam nemo est tam imperitus qui ea quae gessi in consulatu peccata esse dicat, sed nemo est tam inimicus patriae qui non meis consiliis patriam conseruatam esse fateatur.

Voilà donc l'homme que toi, monstrueux fléau, tu as osé appeler exilé, alors que toimême tu es déshonoré par de si grands crimes et par de si grands scandales que tu ne peux te rendre nulle part sans en faire comme un véritable exil ? Qu'est-ce en effet qu'un exilé ? Le nom en lui-même est celui d'un malheur, non d'une infamie. Quand donc l'exil est-il déshonorant ? Dans la réalité quand c'est le châtiment d'un crime, mais de l'avis de tous, même si c'est le châtiment d'une condamnation. Est-ce donc une faute ou un jugement rendu qui me vaut ce nom? Une faute? Désormais tu n'oses plus le dire, toi que tes satellites nomment 'le Catilina qui a réussi', ni aucun de ceux qui avaient coutume de le dire. Il n'y a plus aujourd'hui personne d'assez peu averti pour qualifier de crimes les actes de mon consulat, et il n'y a personne d'assez ennemi de la patrie pour ne pas admettre que la patrie a été sauvée par mes décisions.

13 En l'occurrence, l'analyse de Cicéron ne devait d'ailleurs pas être dénuée de tout fondement juridique : en effet, il évoque longuement, d'abord dans le De domo sua ${ }^{53}$ puis dans le Pro Sestio ${ }^{54}$, un discours prononcé par L. Cotta lors de la séance inaugurale du Sénat, le $1^{\mathrm{er}}$ janvier 57 , et dans lequel est développée l'argumentation suivante: non seulement il n'y avait pas lieu pour le Sénat de voter une loi pour rappeler Cicéron puisque celui-ci, forcé par la violence à quitter Rome, n'avait pas fait l'objet d'une mesure légale d'éloignement, mais encore une telle loi aurait pour effet de légaliser a posteriori une mesure illégale ${ }^{55}$; un sénatus-consulte était donc suffisant. Cicéron ne manquait donc pas d'arguments pour dénoncer la loi de Clodius comme illégale. Pourtant, dans la Correspondance, le discours de Cotta n'est pas même évoqué et la séance du $1^{\mathrm{er}}$ janvier 57 elle-même n'est mentionnée que de façon on ne peut plus laconique, dans deux très courtes lettres à Atticus et une lettre à Q. Cornelius Nepos ${ }^{56}$.

Une telle différence de traitement est bien entendu aisément explicable : tant qu'il est éloigné de Rome, Cicéron ne peut qu'envisager avec réticence la solution proposée par Cotta, dans la mesure où elle aurait pour effet d'ôter à son retour le caractère exceptionnel qu'il entend bien lui donner; en revanche, une fois qu'il est rentré, le discours de Cotta lui fournit un argument juridique non négligeable et il ne manque pas d'en tirer profit: dans la deuxième version que nous avons du discours de Cotta, celle du Pro Sestio, Cicéron prête d'ailleurs à l'orateur des références (comme celle qui est faite au consulat de 63) et des formulations qui valident par avance la présentation qu'il fait de lui-même comme double sauveur de la République ${ }^{57}$. Il n'en reste pas moins que, pour étonnant que cela puisse paraitre, dans ses lettres Cicéron n'aborde jamais de luimême la question de la légalité de la loi de Clodius : elle n'est évoquée qu'en réponse à une lettre d'Atticus qui l'a informé de l'initiative prise en août 58 par Q. Terentius Culleo (un tribun de la plèbe qui lui était favorable) pour la déclarer nulle, exactement comme L. Cotta le propose plus tard ${ }^{58}$. En revanche, comme on l'a depuis longtemps noté, Cicéron n'utilise jamais dans sa correspondance le terme exilium pour définir le 
malheur qui le frappe. Cela tient sans aucun doute en partie à la posture qu'il a choisie dans ses lettres et qui le conduit à privilégier le langage des émotions ${ }^{59}$, mais on peut légitimement se demander, à la lecture des discours, si l'absence du terme exilium n'a pas d'autre explication : soit Cicéron n'a à aucun moment considéré que, juridiquement parlant, son éloignement constituât un exil, soit il a jugé, en prévision de son retour et sans même qu'il soit nécessaire d'imaginer qu'il a déjà élaboré la version qu'il donnerait alors de son départ, qu'il était dangereux pour lui d'employer, dans des lettres susceptibles d'être lues par ses ennemis, un terme qui pourrait ensuite être utilisé comme argument contre lui. Ce n'est certes là qu'une hypothèse, mais il me semble que Cicéron était trop bon connaisseur des lois pour ne pas avoir fait très tôt une telle analyse.

15 La nécessité dans laquelle se trouve Cicéron, à son retour à Rome, de réécrire l'histoire de son départ explique par ailleurs, à mon sens, l'une des spécificités des discours prononcés en 57 et en 56 : je veux parler de l'extrême violence de leur propos, dont l'illustration la plus évidente est le recours répété à l'invective. Cette violence verbale contraste de façon singulière avec le ton, somme toute mesuré, adopté au même moment par Cicéron dans sa correspondance. Clodius y est, il est vrai, régulièrement qualifié de fou (demens) et d'assassin ${ }^{60}$, et les troubles qu'il cause sont constamment dénoncés ${ }^{61}$; mais le laconisme, pour ne pas dire le détachement, avec lequel Cicéron évoque en une formule le discours qu'il a prononcé contre lui au Sénat est très révélateur de sa manière de faire ${ }^{62}$. On objectera non sans raison que l'invective appartient en propre au genre oratoire et que, dans la mesure où elle tire sa force avant tout de l'actio, il n'y a rien d'étonnant à ce que l'on n'en trouve pas dans la Correspondance; mais cela n'explique pas la place qu'elle occupe dans les discours de cette période.

Dans les discours considérés, quatre personnages font l'objet d'invectives. Les deux premiers par ordre d'entrée en scène - puisqu'ils sont attaqués dès le début du Discours de remerciements au Sénat ${ }^{63}$ - sont Pison et Gabinius ${ }^{64}$, les deux consuls de 58. Après avoir été présentés de concert comme indignes de la fonction qui est la leur ${ }^{65}$, ils sont attaqués individuellement ${ }^{66}$. Gabinius est le mignon de Catilina et de bien d'autres, un danseur frisé au petit fer et un marchand de filles (leno); prodigue de son bien et du bien public, il est impie, cruel, débauché, et ivrogne. Pison, « l'étranger transalpin », est pire encore, car il dissimule ses penchants à la volupté sous un air honnête et austère ; il est donc débauché, dissimulé, arrogant et corrompu. Cette liste non limitative de qualificatifs, qui pour être topiques n'en sont pas moins injurieux, est reprise dans le $D e$ domo sua puis dans le Pro Sestio ${ }^{67}$. Le troisième est Sex. Clodius, qui avait été chargé, entre autres choses, de rédiger la deuxième loi de Clodius, celle qui visait directement Cicéron, et qui est dépeint comme «le plus impur des animaux à deux ou à quatre pattes ${ }^{68}$ » et le plus infâme des " dévoreurs de la patrie ${ }^{69}$ ». Le dernier est évidemment Clodius lui-même, "chef suprême des pirates » (archipirata) ambitionnant de contrôler la terre entière ${ }^{70}$, empoisonneur, faussaire, assassin, et sacrilège ${ }^{71}$, mais aussi totalement perdu de mœurs et par-dessus tout incestueux : dans chacun des passages cités Cicéron n'hésite pas en effet à reprendre à son compte contre Clodius et de façon on ne peut plus explicite les couplets obscènes que, dans une lettre à Quintus, il prête à la foule, mais sans les détailler alors qu'il ne s'en prive pas dans les discours ${ }^{72}$. Et si l'attaque ne va parfois pas sans humour, cela n'atténue en rien sa vigueur ${ }^{73}$. 
17 On peut bien entendu penser que Cicéron, contraint à une certaine réserve par les règles du genre épistolaire, profite des discours pour " décharger sa bile ", comme on dit familièrement, contre des personnages qu'il rend collectivement responsables de son malheur et sans doute est-ce une dimension à ne pas négliger. Cicéron est, comme on sait, rancunier ${ }^{74}$ et il a la rancune tenace comme le montre, par sa violence, le discours In Pisonem prononcé en 55. Mais la manière dont sont justifiées dans le Pro Sestio les invectives contre Pison et Gabinius montre que Cicéron, dans le cadre d'une figure rhétorique parfaitement classique de l'éloquence judiciaire - quand on voit la noirceur du personnage, on imagine la noirceur de ses actes -, utilise l'invective en ce qu'elle contribue très efficacement à l'extrême dramatisation de la situation à Rome en 58 (Cic., Sest. 17) :

Sed fuit profecto quaedam illa rei publicae fortuna fatalis, ut ille caecus atque amens tribunus plebis nancisceretur - quid dicam? consules? hocine ut ego nomine appellem euersores huius imperi, proditores uestrae dignitatis, hostis bonorum omnium, qui ad delendum senatum, adfligendum equestrem ordinem, exstinguenda omnia iura atque instituta maiorum se illis fascibus ceterisque insignibus summi honoris atque imperi ornatos esse arbitrabantur? Quorum, per deos immortalis, si nondum scelera uulneraque inusta rei publicae uultis recordari, uultum atque incessum animis intuemini: facilius eorum facta occurrent mentibus uestris, si ora ipsa oculis proposueritis.

C'était sans aucun doute la destinée, pour ainsi dire fatale, de l'État que ce tribun de la plèbe aveugle et fou rencontrât - que dirais-je? des consuls? Est-ce que je peux donner ce nom à des hommes qui ont détruit notre empire, à des traitres à votre dignité, à des ennemis de tous les hommes de bien, qui considéraient que c'était pour détruire le Sénat, pour abaisser l'ordre sénatorial, pour supprimer tous les droits et toutes les institutions de nos ancêtres qu'ils avaient été honorés des faisceaux et de tous les autres insignes de la magistrature et du pouvoir suprêmes? Si vous ne voulez pas encore vous rappeler les crimes, les blessures et les brûlures de la République, représentez-vous leur visage et leur allure: leurs actes vous reviendront plus facilement à l'esprit quand vous verrez de vos yeux leurs traits eux-mêmes.

Une nouvelle fois, le contraste est frappant entre le ton des discours et celui de la Correspondance dans laquelle Cicéron évoque des épisodes de 57 et 56 qui ne sont pas moins violents que ceux de 58 , tout en conservant avec son récit une distance notable et cela même quand les agressions touchent ses proches ${ }^{75}$ ou qu'il en est directement la victime ${ }^{76}$. C'est que la réécriture qui est à l'œuvre rend la dramatisation indispensable : pour que le motif du sacrifice de Cicéron à l'État s'impose, il faut que la République ait été en 58 réellement en danger et de ce point de vue il n'est guère étonnant que Cicéron retrouve dans les discours de cette période les accents des Catilinaires ${ }^{77}$. Ils lui permettent en quelque sorte de «boucler la boucle» et donnent sa cohésion à l'ensemble.

Je voudrais pour conclure insister sur ce dernier point : ce qui est frappant, en effet, dans la réécriture faite par Cicéron dans les discours qu'il prononce à son retour à Rome, des raisons et des conditions de son départ de Rome, donc de ce que nous appelons son «exil », c'est qu'elle est d'une grande cohérence. C'est cette cohérence que fait apparaître, me semble-t-il, la comparaison avec la Correspondance. Il est très intéressant de voir comment s'assemblent peu à peu les éléments d'un discours, au sens littéraire du terme, destiné à lui permettre de sortir grandi de ce qui apparaît a priori comme un épisode fort peu glorieux de sa carrière. C'est ainsi que le motif du sacrifice à la patrie fournit même à Cicéron, ce qui constitue un avantage annexe mais non négligeable, une réponse « toute prête » à l'une des accusations dont il fait l'objet, celle 
d'avoir montré trop de douleur (ou trop peu de philosophie) au moment de quitter Rome. Le fait lui-même était, en l'occurrence, impossible à nier puisque Cicéron avait précisément choisi de multiplier et d'accentuer les manifestations publiques de son abattement (par exemple en parcourant la ville en habits de deuil). Mais pour qui relit les lettres d'exil après les discours, les plaintes de Cicéron prennent une autre tonalité : comment oublier en effet les développements pathétiques du De domo sua ou du Pro Sestio $^{78}$, dans lesquels, loin de nier/renier sa douleur et ses plaintes, l'orateur les revendique comme la preuve même de son sacrifice, puisque l'on ne peut quitter sans souffrir que ce à quoi l'on n'est pas attaché ?

\section{BIBLIOGRAPHIE}

\section{Textes anciens}

Cicero, Back from Exile. Six Speeches upon His Return, transl. with introd. and notes by D. R. Shackleton Bailey, Classical Resources Series 4, Chicago.

\section{Textes modernes}

GARCEA A. 2005, Cicerone in esilio. L'epistolario e le passioni, Spudasmata 103, Hildesheim - New York.

\section{NOTES DE FIN}

1. C'est un point sur lequel je reviendrai.

2. Si la première partie de cette formule très rhétorique renvoie de façon générale à l'éloquence judiciaire, la deuxième partie est une allusion claire aux événements de 63 et à la condamnation à mort des complices de Catilina.

3. Cic., Att. 4, 1, 3: Nos adhuc in nostro statu quod difficillime recuperari posse arbitrati sumus, splendorem nostrum illum forensem et in senatu auctoritatem et apud uiros bonos gratiam, magis quam opta<ra>mus consecuti sumus. Cette lettre, datée de la mi-septembre, est la première de celles qu'il écrit après son retour.

4. Cic., Att. 4, 1, 5-6.

5. Il s'agit très probablement du discours de remerciements que nous connaissons sous le titre Cum populo gratias agit. C'est la conclusion à laquelle conduit le passage du De domo sua (Dom. 7) où sont évoqués les événements du 7 septembre.

6. Cic., Att. 4, 2, 2 : Post illas datas litteras secuta est summa contentio de domo. diximus apud pontifices prid. Kal. Oct. acta res est accurate a nobis, et, si umquam in dicendo fuimus aliquid aut etiam si numquam alias fuimus, tum profecto dolor et <rei> magnitudo uim quandam nobis dicendi dedit. itaque oratio iuuentuti nostrae deberi non potest; quam tibi, etiam si non desideras, tamen mittam cito, "Après que j'eus écrit cette lettre [celle de la mi-septembre, Att. 4, 1], éclata le grand conflit au sujet de ma maison. J'ai parlé devant les pontifes le 29 septembre. J'ai traité la question avec grand soin et si 
j'ai eu jamais quelque talent comme orateur ou même si je n'en ai jamais eu, alors sans aucun doute mon ressentiment et l'importance de la question m'ont donné une sorte de puissance oratoire. C'est pourquoi il n'est pas possible de priver la jeunesse de ce discours; je vais donc te l'envoyer rapidement, même si tu ne le veux pas. » On notera que ce jugement enthousiaste n'est pas partagé par la majorité des spécialistes, qui ne classent pas le discours parmi les meilleurs de ceux qu'a prononcés Cicéron.

7. Cic., Q. fr. 2, 1, 3 : Postea Racilius de priuatis me primum sententiam rogauit. Multa feci uerba de toto furore latrocinioque P. Clodi; tamquam reum accusaui multis et secundis admurmurationibus cuncti senatus. Orationem meam collaudauit satis multis uerbis non mehercule indiserte Vetus Antistius, isque iudiciorum causam suscepit antiquissimamque se habiturum dixit. Pour les accusations lancées à cette occasion contre Clodius, voir ci-dessous.

8. Cic., Fam. 1, 2, 1 : Idibus Ianuariis in senatu nihil est confectum propterea quod dies magna ex parte consumptus est altercatione Lentuli consulis et Canini tr<ibuni> pl. Eo die nos quoque multa uerba fecimus maximeque uisi sumus senatum commemoratione tuae uoluntatis erga illum ordinem permouere.

9. Cic., Q. fr. 2, 3, 6 : A. d. iii Id. Febr. dixi pro Bestia de ambitu apud praetorem Cn. Domitium in foro medio maximo conuentu incidique in eum locum in dicendo cum Sestius multis in templo Castoris uulneribus

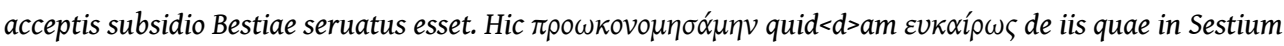
apparabantur crimina et eum ornaui ueris laudibus magno adsensu omnium.

10. Cic., Q. fr. 2, 4, 1 : Sestius noster absolutus est a. d. ii Id. Mart. et, quod uehementer interfuit rei publicae, nullam uideri in eius modi causa dissensionem esse, omnibus sententiis absolutus est. Illud quod tibi curae saepe esse intellexeram, ne cui iniquo relinqueremus uituperandi locum, qui nos ingratos esse diceret nisi illius peruersitatem quibusdam in rebus quam humanissime ferremus, scito hoc nos in eo iudicio consecutos esse ut omnium gratissimi iudicaremur. Nam defendendo moroso homini cumulatissime satis fecimus et, id quod ille maxime cupiebat, Vatinium, a quo palam oppugnabatur, arbitratu nostro concidimus dis hominibusque plaudentibus. L'Interrogatio in Vatinium est directement liée au Pro Sestio puisque Vatinius avait été cité comme témoin à charge au procès de ce dernier.

11. Cic., Q.fr. 2, 4a, 4: «Dans les procès, je suis ce que j'étais : ma maison est remplie comme jamais. »

12. Cic., Q. fr. 2, 4a, 4 : Vnum accidit <im>prudentia Milonis incommodum de Sex. Clodio, quem neque hoc tempore neque ab imbecillis accusatoribus mihi placuit accusari. Ei tres sententiae deterrimo in consilio defuerunt. Itaque hominem populus reuocat et retrahatur necesse est. Non enim ferunt homines et, quia cum apud suos diceret paene damnatus est, uident damnatum. Ea ipsa in re Pompei offensio nobis obstitit. senatorum enim urna copiose absoluit, equitum adaequauit, tribuni aerarii condemnarunt. Sed hoc incommodum consolantur cottidianae damnationes inimicorum, in quibus me perlibente Seuius adlisus est, ceteri conciduntur. L'exemple du procès contre Sextus Clodius montre ainsi que c'est Milon qui avait monté l'accusation contre ce personnage.

13. Cic., Att. 4, 5, 1 : Ain tu? An me existimas ab ullo malle mea legi probarique quam $<a>$ te ? cur igitur cuiquam misi prius? urgebar ab eo ad quem misi et non habebam exempla duo. quin etiam (<iam>dudum enim circumrodo quod deuorandum est) subturpicula mihi uidebatur esse $\pi \alpha \lambda \imath v \omega \delta i ́ \alpha$.

14. Voir Cic., Dom.9-31. Si l'on en croit le discours, Clodius reprochait à Cicéron d'avoir fait conférer à Pompée des pouvoirs extraordinaires. Ce qui est vrai ; mais la lettre à Atticus (Att. 4, 1, 6-7) dans laquelle la question est évoquée présente la motion de Cicéron d'une part comme répondant aux demandes du peuple, lequel est, selon lui, largement instrumentalisé par Clodius, d'autre part comme destinée à contrer une motion, celle de Messius, qui aurait donné à Pompée des pouvoirs encore plus étendus que ceux qu'il a finalement obtenus.

15. Les haruspices avaient en effet expliqué les prodiges qui s'étaient produits dans le Latium et sur lesquels le Sénat les avait consultés comme le signe de la colère des dieux et avaient, entre autres, donné comme raison à cette colère «la profanation de lieux sacrés ». Or on sait que Clodius avait consacré une partie du terrain de Cicéron en y plaçant une statue de Libertas.

16. Cic., Fam. 5, 12, 6.

Interférences, 8 | 2015 
17. C'est pour cette raison qu'ils ont été réunis en 1991 par D. R. Schackleton Bailey, qui en a proposé une traduction (avec quelques notes) sous le titre: Cicero-Back from Exile. Six Speeches upon His Return.

18. La Correspondance témoigne largement de l'aide que Sestius apporte à Cicéron même si celui-ci n'approuve pas toujours ses initiatives: voir par ex. Q.fr. 1, 4; Att.3, 20 et Att. 3, 23, où il est question d'une rogatio qu'il a déposée et qui ne plaît pas à Cicéron; Att. 4, 3 où il souligne sa présence lors des comparutions de Milon devant le peuple...

19. On a souvent dit que, dans ce discours, Cicéron consacrait fort peu de place à Sestius luimême. Mais il faut tenir compte du fait que la cause de Sestius avait été plaidée sur le fond par Hortensius (Cic., Sest. 3) avant que Cicéron ne prenne la parole.

20. Cic., Dom. 32.

21. Cic., Dom. 100-141.

22. Fam. 14, 3, 1 - aux siens: Meum fuit officium uel legatione uitare periculum uel diligentia et copiis resistere uel cadere fortiter.

23. Cic., Q. fr. 1, 4, 1: Amabo te, mi frater, ne, si uno meo facto et tu et omnes mei corruistis, improbitati et sceleri meo potius quam imprudentiae miseriaeque adsignes. Nullum est meum peccatum nisi quod is credidi a quibus nefas putarem esse me decipi aut etiam quibus ne id expedire quidem arbitrabar. Intimus, proximus, familiarissimus quisque aut sibi pertimuit aut mihi inuidit. Ita mihi nihil misero praeter fidem amicorum, cautum meum consilium, <de>fuit. Voir aussi Att. 3, 13, 2: nam quod scribis te audire me etiam mentis errore ex dolore adfici, mihi uero mens integra est. atque utinam tam in periculo fuisset, cum ego iis quibus meam salutem carissimam esse arbitrabar, inimicissimis crudelissimisque usus sum ! qui ut me paulum inclinari timore uiderunt, sic impulerunt ut omni suo scelere et perfidia abuterentur ad exitium meum.

24. Cic., Fam. 14, 1, 2: Quod si nostris consiliis usi essemus neque apud nos tantum ualuisset sermo aut stultorum amicorum aut improborum, beatissimi niueremus.

25. Cic., Att. 3, 15, 4 : Sed tu tantum lacrimas praebuisti dolori meo, quod erat amoris, tamquam ipse ego ; quod meritis meis perfectum potuit, ut dies ac noctes quid mihi faciendum esset cogitares, id abs te meo, non tuo scelere praetermissum est. Quod si non modo tu sed quisquam fuisset qui me Pompei minus liberali responso perterritum a turpissimo consilio reuocaret, quod unus tu facere maxime potuisti, <aut honeste occubuissemus > aut uictores hodie uineremus.

26. Cic., Att. 3, 1 et 3, 3.

27. Cic., Fam. 14, 2, 1: Vos enim uideo miserrimas, quas ego beatissimas semper esse uolui, idque praestare debui et, nisi tam timidi fuissemus, praestitissem.

28. Cic. Att. 3, 15, 5 : Nam prior lex nos nihil laedebat. Quam si, ut est promulgata, laudare uoluissemus aut, ut erat neglegenda, neglegere, nocere omnino nobis non potuisset. Hic mihi primum meum consilium defuit, sed etiam obfuit. Caeci, caeci inquam, fuimus in uestitu mutando, in populo rogando, quod, nisi nominatim mecum agi coeptum esset, fieri perniciosum fuit.

29. Cic., Att. 3, 13, 7 : Nunc, Pomponi, quoniam nihil impertisti tuae prudentiae ad salutem meam, quod aut in me ipso satis esse consili decreras aut te nihil plus mihi debere quam ut praesto esses, quoniamque ego proditus, inductus, coniectus in fraudem omnia mea praesidia neglexi, totam Italiam [in me] erectam ad me defendendum destitui et reliqui, me, meos tradidi inimicis inspectante et tacente te, qui, si non plus ingenio ualebas quam ego, certe timebas minus: si potes, erige adflictos et in eo nos iuua.

30. Cic., Fam. 14, 3, 1. L'expression turpissimum consilium se trouve en Att. 3, 15, 4 (texte cité note 25). Sur la question de la prise de décision et de sa présentation dans les lettres d'exil, voir dans le présent volume la contribution de Ch. Guérin : «Délibération, conseil et prise de décision dans les lettres d'exil de Cicéron ».

31. Cic., Red. Sen. 4 : « cette année dont j'ai préféré qu'elle fût fatale pour moi plutôt que pour la patrie ». L'emploi de malueram est, à lui seul, la marque du changement de présentation des faits.

32. Cic., Red. Sen. 32-34.

33. L'argument revient, très significativement, à deux reprises (Red. Sen. 32 et 34). 
34. Cic., Red. Sen. 34 : Sed, cum uiderem me non diutius quam ipsam rem publicam ex hac urbe afuturum, neque ego, illa exterminata, mihi remanendum putaui, et illa, simul atque reuocata est, me secum pariter reportauit, «Mais comme je voyais que je ne serais pas absent de notre ville plus longtemps que la République elle-même, pour ma part j'ai pensé qu'il ne fallait pas que j'y reste alors qu'elle en était expulsée ; et elle, dès qu'elle a été rappelée, m'a ramené avec elle ».

35. Cic., Att. 4, 1, 1: Cognoram enim, ut uere scribam, te in consiliis mihi dandis nec fortiorem nec prudentiorem quam me ipsum nec etiam propter meam in te obseruantiam nimium in custodia salutis meae diligentem; eundemque te, qui primis temporibus erroris nostri aut potius furoris particeps et falsi timoris socius fuisses, acerbissime discidium nostrum tulisse plurimumque operae, studi, diligentiae, laboris ad conficiendum reditum meum contulisse.

36. Voir Cic., Att. 2, 19, 4 : Noster autem Publius mihi minitatur, inimicus est. Impendet negotium, ad quod tu scilicet aduolabis. Videor mihi nostrum illum consularem exercitum bonorum, etiam satis bonorum habere firmissimum. - Att. 2, 22, 3 : Domus celebratur, occurritur, renouatur memoria consulatus, studia significantur; in eam spem addicimur ut nobis ea contentio quae impendet interdum non fugienda uideatur. - Q. fr. 1, 2, 16 : Nostrae tamen causae non uidentur homines defuturi; mirandum in modum profitentur, offerunt se, pollicentur. Equidem cum spe sum maxima tum maiore etiam animo: spe, ut superiores fore nos confidam; animo, ut in hac re publica ne casum quidem ullum pertimescam. Sed tamen se res sic habet : si diem nobis dixerit, tota Italia concurret, ut multiplicata gloria discedamus; sin autem ui agere conabitur, spero fore studiis non solum amicorum sed etiam alienorum ut ui resistamus. Omnes et se et suos amicos, clientis, libertos, seruos, pecunias denique suas pollicentur. Nostra antiqua manus bonorum ardet [et] studio nostri atque amore. Si qui antea aut alieniores fuerant aut languidiores, nunc horum regum odio se cum bonis coniungunt.

37. Cic., Vat. 6 : Nam quod mihi discessum obiecisti meum, et quod horum, quibus ille dies acerbissimus fuit qui idem tibi laetissimus, luctum et gemitum renouare uoluisti, tantum tibi respondeo me, cum tu ceteraeque rei publicae pestes armorum causam quaereretis, et cum per meum nomen fortunas locupletium diripere, sanguinem principum ciuitatis exsorbere, crudelitatem uestram odiumque diuturnum quod in bonos iam inueteratum habebatis saturare cuperetis, scelus et furorem uestrum cedendo maluisse frangere quam resistendo.

38. De ce point de vue, le fait que Cicéron se place sous le regard des yeux immortels, comme le général qui se dévoue, ne fait qu'accentuer le détournement du rituel.

39. D'autant que, si, comme c'est très vraisemblable, le discours que nous lisons a fait l'objet de corrections avant d'être publié, le maintien du terme deuotio relève d'un choix délibéré de la part de Cicéron.

40. Cic., Dom. 145 : [...] si in illo paene fato rei publicae obieci meum caput pro uestris caerimoniis atque templis perditissimorum ciuium furori atque ferro, et si iterum, cum ex mea contentione interitus bonorum omnium quaereretur, uos sum testatus, uobis me ac meos commendaui, meque atque meum caput ea condicione deuoui ut, si et eo ipso tempore et ante in consulatu meo commodis meis omnibus, emolumentis, praemiis praetermissis cura, cogitatione, uigiliis omnibus nihil nisi de salute meorum ciuium laborassem, tum mihi re publica aliquando restituta liceret frui, sin autem mea consilia patriae non profuissent, ut perpetuum dolorem auulsus a meis sustinerem, hanc ego deuotionem capitis mei, cum ero in meas sedis restitutus, tum denique conuictam esse et commissam putabo.

41. P. Decius Mus s'était dévoué aux dieux infernaux en 340, lors de la bataille de Véséris, contre les Latins. Son fils avait imité son exemple en 295 à la bataille de Sentinum où les Romains affrontaient les Gaulois et les Samnites.

42. Cic., Dom. 64 : Videbam uiuo senatu populoque Romano celerem mihi summa cum dignitate reditum, nec intellegebam fieri diutius posse ut mihi non liceret esse in ea re publica quam ipse seruassem. Quod si non liceret, audieram et legeram clarissimos nostrae ciuitatis uiros se in medios hostis ad perspicuam mortem pro salute exercitus iniecisse: ego pro salute uniuersae rei publicae dubitarem hoc meliore condicione esse quam Decii, quod illi ne auditores quidem suae gloriae, ego etiam spectator meae laudis esse 
potuissem? Itaque infractus furor tuus inanis faciebat impetus; omnem enim uim omnium sceleratorum acerbitas mei casus exceperat; non erat in tam immani iniuria tantisque ruinis nouae crudelitati locus.

43. Cic., Sest. 45-49.

44. La situation qu'il envisage est celle du passager d'un navire attaqué par les pirates qui demandent qu'on le leur livre et se jetant de lui-même dans les flots pour sauver la vie de tous les autres.

45. Cic., Sest. 48 : ex qua <cuiuitate> P. Decius primum pater, post aliquot annos patria uirtute praeditus filius se ac uitam suam instructa acie pro salute populi Romani uictoriaque deuouisset.

46. Cic., Sest. 49 : Haec ego et multa alia cogitans hoc uidebam, si causam publicam mea mors peremisset, neminem umquam fore qui auderet suscipere contra improbos ciuis salutem rei publicae; itaque non solum si ui interissem, sed etiam si morbo exstinctus essem, fore putabam ut exemplum rei publicae conseruandae mecum simul interiret. Quis enim umquam me a senatu populoque Romano tanto omnium bonorum studio non restituto - quod certe, si essem interfectus, accidere non potuisset - ullam rei publicae partem cum sua minima inuidia auderet attingere ? Seruaui igitur rem publicam discessu meo, iudices [...].

47. Cic., Dom. 76 : Vno enim maledicto bis a me patriam seruatam esse concedis : semel, cum id feci quod omnes non negant immortalitati, si fieri potest, mandandum, tu supplicio puniendum putasti, iterum, cum tuum multorumque praeter te inflammatum in bonos omnis impetum meo corpore excepi, ne eam ciuitatem quam seruassem inermis armatus in discrimen adducerem, «En effet, par une seule injure tu conviens que j'ai sauvé deux fois la patrie : la première fois quand j'ai fait ce qui, de l'avis de tous, mériterait l'immortalité, si c'était chose possible, et que toi tu as jugé digne de supplice; la deuxième fois quand j'ai supporté sur ma personne l'assaut impétueux que tu menais contre tous les gens de bien, toi et la foule des tiens, afin d'épargner à la cité que j'avais sauvée sans recourir aux armes le danger qu'elle aurait couru si je les avais pris ».

48. Cic., Sest. 49. Gloria renvoie au consulat de 63 et aerumna au départ de Cicéron en 58.

49. Cic., Dom. 26 : Extra ordinem ferri nihil placet Clodio. Quid? de me quod tulisse te dicis, patricida, fratricida, sororicida, nonne extra ordinem tulisti? An de peste ciuis, quemadmodum omnes iam di atque homines iudicarunt, conseruatoris rei publicae, quem ad modum autem tute ipse confiteris, non modo indemnati sed ne accusati quidem, licuit tibi ferre non legem sed nefarium priuilegium, lugente senatu, maerentibus bonis omnibus, totius Italiae precibus repudiatis, oppressa captaque re publica. Pour l'invective contre Clodius, voir plus loin.

50. Cic., Dom.43-50. On trouve une autre analyse de la loi, infiniment moins détaillée, en Cic., Sest. 65.

51. Voir en particulier Cic., Dom. 47 sur la formule ut interdictum sit, qui semble impliquer que l'interdiction de l'eau et du feu avait été faite a posteriori. Je renvoie sur cette question à la contribution de M. Ducos dans ce volume.

52. Cic., Dom. 62 : Nihil erat latum de me; non adesse eram iussus, non citatus afueram; eram etiam tuo iudicio incolumis...

53. Cic., Dom. 68.

54. Cic., Sest. 73.

55. Je résume ici Cic., Dom. 68.

56. Cic. Att. 3, 26 ; Att. 3, 27 ; Fam. 5, 4.

57. Cic., Sest. 73 : Tum princeps rogatus sententiam L. Cotta dixit id quod dignissimum re publica fuit, nihil de me actum esse iure, nihil more maiorum, nihil legibus; non posse quemquam de ciuitate tolli sine iudicio ; de capite non modo ferri, sed ne iudicari quidem posse nisi comitiis centuriatis; uim fuisse illam, flammam quassatae rei publicae perturbatorumque temporum; iure iudiciisque sublatis, magna rerum permutatione impendente, declinasse me paulum et spe reliquae tranquillitatis praesentis fluctus tempestatemque fugisse; qua re, cum absens rem publicam non minus magnis periculis quam quodam tempore praesens liberassem, non restitui me solum sed etiam ornari a senatu decere. Les deux versions que donne Cicéron du discours de Cotta apparaissent comme très proches l'une de l'autre comment d'ailleurs pourrait-il en être autrement puisque le discours avait été prononcé devant 
le Sénat? - mais en réalité la version donnée dans le Pro Sestio trahit clairement une réécriture par rapport à celle que l'on trouve dans le De domo sua.

58. Cic., Att. 3, 15, 5 du 17 août 58.

59. Voir sur ce point Garcea 2005.

60. Voir par ex. Cic., Att. 4, 3, 2.

61. Voir par ex. Cic., Q. fr. 2, 1, 3.

62. Cic., Q. fr. 2, 1, 3 : multa feci uerba de toto furore latrocinioque P. Clodi; tamquam reum accusaui multis et secundis admurmurationibus cuncti senatus.

63. Cic., Red. Sen. 10-19.

64. On notera au passage qu'il n'y a aucune attaque contre ces deux personnages dans la Correspondance: tout juste Cicéron se réjouit-il que Gabinius se soit vu refuser par le Sénat les «supplications » qu'il avait demandées pour sa campagne contre les Arabes. Voir Cic., Q. fr. 2, 6, 1. 65. Cic., Red. Sen. 10.

66. Cic., Red. Sen. 11-18.

67. Voir Cic., Dom. 23 et 62 ; Sest. 18-25.

68. Cic. Dom. 48: Hoc tu scriptore, hoc consiliario, hoc ministro, omnium non bipedum sed etiam quadripedum impurissimo, rem publicam perdidisti.

69. Voir Cic., Sest. 25-26 pour l'expression helluo patriae. Voir aussi Dom. 25 : Scilicet tu helluoni spurcatissimo, praegustatori libidinum tuarum, homini egentissimo et facinerosissimo, Sex. Clodio, socio tui sanguinis, qui sua lingua etiam sororem tuam a te abalienauit, omne frumentum priuatum et publicum, omnis prouincias frumentarias, omnis mancipes, omnis horreorum clauis lege tua tradidisti.

70. Cic., Dom. 24.

71. Cic., Sest. $17 ; 39 ; 95$; Har. 31-39.

72. Cic., Q. fr. 2, 3, 2 : ea res acta est, cum hora sexta uix Pompeius perorasset, usque ad horam octauam, cum omnia maledicta, uersus denique obscenissimi in Clodium et Clodiam dicerentur.

73. Cic., Dom. 92 : Hic tu me etiam gloriari uetas; negas esse ferenda quae soleam de me praedicare, et homo facetus inducis etiam sermonem urbanum ac uenustum, me dicere solere esse me Iouem, eundemque dictitare Mineruam esse sororem meam. Non tam insolens sum, quod Iouem esse me dico, quam ineruditus, quod Mineruam sororem Iouis esse existimo; sed tamen ego mihi sororem uirginem adscisco, tu sororem tuam uirginem esse non sisti. Sed uide ne tu te soleas Iouem dicere, quod tu iure eandem sororem et uxorem appellare possis, «Mais voici que tu me défends aussi de me glorifier; tu dis que mes vantardises habituelles ne sont pas tolérables et en homme spirituel tu emploies un langage plaisant et délicat : tu dis que je me prends pour Jupiter et que je répète que Minerve est ma sœur. Je suis moins orgueilleux quand je dis que je suis Jupiter, qu'ignorant quand je pense que Minerve est sa sœur. Mais moi au moins c'est une vierge que je prends pour sœur, alors que toi tu n'as pas laissé ta sœur rester vierge. Prends garde, toi, à ne pas te prendre pour Jupiter, puisque tu peux à bon droit appeler la même femme ta sœur et ton épouse. »

74. Il enrage par ex. ( $Q$. fr. 2, 4a, 4) de constater que Milon n'a pas réussi, parce qu'il a mal monté l'accusation, à faire condamner Sex. Clodius.

75. C'est le cas, par ex., de l'attaque en plein jour de la maison de Milon par les bandes clodiennes, qui donne lieu avec l'intervention de Q. Flaccus à une véritable bataille rangée dans laquelle périssent de nombreux partisans de Clodius.

76. L'exemple le plus révélateur en est, par l'humour dont il fait preuve, le récit de l'agression dont il a été victime sur la voie sacrée en novembre 57 : Itaque ante diem tertium Idus Nouembres, cum Sacra uia descenderem, insecutus est me cum suis. clamor, lapides, fustes, gladii ; et haec improuisa omnia. discessi in uestibulum Tetti Damionis. qui erant mecum facile operas aditu prohibuerunt. ipse occidi potuit; sed ego diaeta curare incipio, chirurgiae taedet. ille omnium uocibus cum se non ad iudicium sed ad supplicium praesens trudi uideret, omnis Catilinas Acidinos postea reddidit (Cic., Att. 4, 3, 3).

77. Entre autres exemples, voir Cic., Dom. 96 : Cum omnium perditorum et coniuratorum incitata uis, duce tribuno plebis, consulibus auctoribus, adflicto senatu, perterritis equitibus Romanis, suspensa ac 
sollicita tota ciuitate, non tam in me impetum faceret quam per me in omnis bonos, me uidisse, si uicissem, tenuis rei publicae reliquias, si uictus essem, nullas futuras. Quod cum iudicassem, defleui coniugis miserae discidium, liberorum carissimorum solitudinem, fratris absentis amantissimi atque optimi casum, subitas fundatissimae familiae ruinas; sed his omnibus rebus uitam anteposui meorum ciuium, remque publicam concidere unius discessu quam omnium interitu occidere malui. Speraui, id quod accidit, me iacentem posse uiuis uiris fortibus excitari; si una cum bonis interissem, nullo modo posse <rem publicam> recreari.

78. Cic., Dom. 97-98; Sest. 49.

\section{RÉSUMÉS}

Rompant radicalement avec la présentation qu'il fait dans sa Correspondance de son départ de Rome comme une faute ou une erreur, Cicéron affirme dans ses discours qu'il a délibérément choisi de quitter la Ville pour sauver la République en enlevant à ceux qui voulaient la détruire tout prétexte pour y répandre la violence armée. Autour du motif de la deuotio, il construit au fil des discours une version des événements d'une grande cohérence et qui est destinée à lui permettre non seulement de retrouver son statut, mais de sortir grandi d'une période très difficile de sa carrière.

In his letters Cicero presents his departure from Rome as a fault or an error. In his speeches on the contrary he says he voluntarily chose to leave Rome to save the Republic threatened by the attacks of his enemies. Around the motif of sacrifice (deuotio), he methodically built a version of events intended to allow him to leave up his period of "exile".

\section{INDEX}

Keywords : culpa/error, exilium, deuotio, invective, letters, speeches, dramatization of the story Mots-clés : culpa/error, exilium, deuotio, invectives, lettres, discours, dramatisation du récit nomsmotscles Cicéron

\section{AUTEUR}

\section{GÉRARD SALAMON}

ENS de Lyon, HiSoMA (UMR 5189) 